\section{EPV016/\#272 RESILIENCE IN BREAST CANCER PATIENTS AND ITS ASSOCIATION WITH ANXIETY AND DEPRESSION}

${ }^{1} S$ Sghaier*, ${ }^{1} \mathrm{M}$ Bouhani, ${ }^{2} \mathrm{M}$ Slimane, ${ }^{2} \mathrm{M}$ Bouida, ${ }^{3} \mathrm{~N}$ Bram, ${ }^{2} \mathrm{~K}$ Rahal. ${ }^{1}$ Salah Azaiez Institute, Surgical Oncology Department, Tunis, Tunisia; ${ }^{2}$ Salah Azaiez Institute of Oncology, Surgical Oncology, Tunis, Tunisia; ${ }^{3}$ Razi Hospital, Freud Department, Tunis, Tunisia

\subsection{6/ijgc-2021-IGCS.83}

Objectives Breast Cancer is a stressful event. Several breast cancer patients may suffer from clinically relevant symptoms of depression and/or anxiety. Indeed,resilience represents an important psychosocial factor. We aim to report the resilience of breast cancer women and its association with depression and anxiety.

Methods It is a cross-sectional descriptive study conducted over a third-month period, from January 2nd, 2020 to March 30th, 2020 at Salah Azaiez Institute including 61 breast cancer patients.

Results The median age was 55.7 years. Forty-six percent were housewives. The illiteracy rate was $34.4 \%$. The average duration of marriage was 27.8 years.Co-morbidities were found in $36.1 \%$ of patients. The average time after treatment was 60.8 months.57.4\% of patients were classified as stage II. Radical surgery was conducted in $70.5 \%$ of patients. Chemotherapyand Hormone therapy were administered in $90.2 \%$ and $83.6 \%$ of cases, respectively. The average score of resilience (RS-14) was de 76.6. The average scores of anxiety and depression were10.5 and 8.6, respectively.Clinically relevant symptoms of anxiety and depression were reported with $52.5 \%$ and $29.5 \%$ of patients, respectively. Correlation analysis showed that the RS-14 and anxiety and depression of the HAD scale were in negative correlation $(r=-0.419, \mathrm{p}<0.001)$ and $(r=-0.606, p<0.001)$, respectively. Predictor factors of resilience were co-morbidities and not receiving hormone therapy.

Conclusions Resilience represents a protective psychosocial factor against anxiety and depression among breast cancer patients. A multidisciplinary healthcare team is crucial for patient psychosocial care throughout the cancer continuum allowing to promote resilience and leading to enhance the quality of life.

\section{EPV017/\#299 PHYLLODES TUMORS OF THE BREAST: A RETROSPECTIVE ANALYSIS OF CLINICOPATHOLOGICAL FEATURES OF LOCAL RECURRENCE}

${ }^{1} \mathrm{H}$ Bouaziz, ${ }^{2} \mathrm{~N}$ Tounsi, ${ }^{2} \mathrm{~S}$ Jouini, ${ }^{2} \mathrm{~S} S \mathrm{Sakhri,}{ }^{3} \mathrm{~S}$ Yahiaoui, ${ }^{4} \mathrm{~S}$ Kammoun, ${ }^{2} \mathrm{~K}$ Rahal. ${ }^{1}$ Salah Azaiez Institute of Oncology, Surgical Oncology, Tunis, Tunisia; ${ }^{2}$ Salah Azaiez Institute of Oncology, Surgical Oncology, Tunis, Tunisia; ${ }^{3}$ Salah Azaiez Institute of Oncology, Radiotherapy, Tunis, Tunisia; ${ }^{4}$ Salah Azaiez Institute of Oncology, Department of Pathology, Tunis, Tunisia

\subsection{6/ijgc-2021-IGCS.84}

Objectives Phyllodes tumors are biphasic tumors consisting of epithelial and stromal components that account for less than $1 \%$ of all breast tumors.

Methods Forty-one cases of phyllodes tumor (borderline and phyllode sarcoma) diagnosed between (2010-2018) were retrospectively studied. Cox proportional hazards regression models were used to analyze associations between clinicopathological features and disease recurrence $\mathrm{P}<0.05$ was considered significant.

Results From 41 tumors diagnosed 19 cases were classed as borderline $(46.3 \%)$ and malignant phyllodes tumors in 22 cases $(53.7 \%)$. Twenty-three $(56.1 \%)$ were treated with mastectomy and 18 (43.9\%) with conservative surgery. Seventeen (41.4\%) of patient underwent adjuvant radiotherapy and three patients received chemotherapy. During a median follow-up of 80 months, LR rate was $12.2 \%$, and $9.8 \%(\mathrm{p}=$ 0.4 ) respectively in the borderline, and malignant groups. Young age ( $<35$ years), presence of necrosis and positive surgical margin, severe stromal atypia, severe versus mild stromal cellularity, higher index mitosis was associated with increasing risk factor for LR. However only severe stromal cellularity was associated with significant prognostic factor (p: 0.005) in our study.

Conclusions Multiple histopathological features influence phyllodes recurrence.

\section{EPV018/\#301 MALIGNANT PHYLLODES TUMORS OF THE BREAST: PATTERNS OF CARE AND PREDICTORS OF ADJUVANT RADIATION THERAPY}

${ }^{1} \mathrm{H}$ Bouaziz, ${ }^{2} \mathrm{~N}$ Tounsi*, ${ }^{2} \mathrm{~S}$ Jouini, ${ }^{1} \mathrm{M}$ Ghalleb, ${ }^{3} \mathrm{~S}$ Yahiaoui, ${ }^{4} \mathrm{~S}$ Kammoun, ${ }^{1} \mathrm{~K}$ Rahal. ${ }^{\text {SSalah }}$ Azaiez Institute of Oncology, Surgical Oncology, Tunis, Tunisia; ${ }^{2}$ Salah Azaiez Institute of Oncology, Surgical Oncology, Tunis, Tunisia; ${ }^{3}$ Salah Azaiez Institute of Oncology, Radiotherapy, Tunis, Tunisia; ${ }^{4}$ Salah Azaiez Institute of Oncology, Department of Pathology, Tunis, Tunisia

\subsection{6/ijgc-2021-IGCS.85}

Objectives The role of adjuvant radiation therapy RT in Malignant Phyllodes Tumors of the Breast MPTB is still controversial Our objective was to investigate the role of adjuvant (RT) in MPTB.

Methods Patients diagnosed with MPTB and treated at our institution from January 2010 to December 2019 were reviewed.

Results A total of 23 patients with non metastatic MPTB were included in our study. The median age was 41 years old (18-70 years). All patients underwent primary surgical treatment. Four patients had conserving surgery with clear surgical margins. Definitive mastectomy was carried out in 19 patients (82\%). The median pathological tumor size was $12 \mathrm{~cm}(3-25 \mathrm{~cm})$. Sixteen patients had adjuvant RT. Only one patient with breast conserving surgery had adjuvant RT. Fifteen patients who were treated with mastectomy had adjuvant RT with delivering a dose of 50Gy in 25 fractions over 5 weeks. Adjuvant RT was more indicated for patients with heterologous component (4/7), tumors $\geq 5 \mathrm{~cm}$ (14/20); presence of high atypia (15/19),tumoral necrosis (4/5), high mitoses $(10 / 13)$. Unfortunately, five patients had local recurrence LR, from them two patients had adjuvant radiotherapy $(p=0.1)$. In addition, four patient had distant metastases, from them tow had radiotherapy $(p=0.3)$. The median overall survival was 180 months. Adjuvant RT was not independently associated with improvement in overall survival $(\mathrm{P}=$ $0.6)$.

Conclusions The benefit of Adjuvant radiotherapy for MPTB was not yet clear. Further study was needed to establish the real place of adjuvant RT. 